\title{
Defensive use of publications in an intellectual property strategy
}

\author{
Although it has been slow to catch on in biotechnology, defensive publication can be an \\ important part of your comprehensive IP strategy.
}

Bill Barrett

A search of the US Patent database from 1996 to 2001 reveals almost 10,000 patents that cite the IBM Technical Disclosure Bulletin as prior art $^{1}$. The Bulletin, which is published as a component of Research Disclosure, is a mechanism for defensive publishing. Citation of a publication, such as the Bulletin, in a patent application indicates that the publication contributes to the state of the art against which the application is judged. IBM uses the Bulletin to advance the state of the art, thereby raising the bar for its competitors' patent applications. In other words, Bulletin publications force IBM's competitors to narrow their patent claims, helping IBM to reduce the possibility that its competitors' patent claims will encompass IBM inventions.

Citation of such a large number of Bulletin articles strongly suggests that IBM's defensive publication strategy is paying off. Despite its successful use by IBM and a handful of other companies, defensive publication has been slow to catch on as an intellectual property (IP) strategy in biotechnology. This article discusses the legal implications of defensive publications, analyzes strategic considerations for determining whether to publish, patent, or maintain an invention as a trade secret, discusses legal considerations for the preparation of defensive publications, and discusses forums for defensive publication.

\section{What are defensive publications?}

The heart of the patent system is a balanced deal between the government (representing the public) and the patent applicant, and an understanding of this deal sets the context for a discussion of defensive publication. The deal requires the inventor to disclose a new invention to the public. In exchange, the government grants the inventor a temporary monopoly on his or her new invention. The purpose of a defensive publication is to destroy the balance of

Bill Barrett is an intellectual property consultant at ipCapital Group, 400 Cornerstone Drive, Suite 325, Williston, VT 05495 (bbarrett@ipcapitalgroup.com). the deal by disclosing an invention to the public. If the defensive publication predates the filing of the inventor's patent application, the patent deal for that application is out of balance: the patent applicant has nothing new to disclose to the public, because the invention has already been disclosed and is thus already possessed by the public. Technically speaking, the successful defensive publication renders the competitor's invention obvious or lacking in novelty.

There is a caveat to the general principle that defensive publications destroy patent rights. In the United States, a competitor may overcome a defensive publication by presenting evidence showing a date of conception that precedes the date of the defensive publication. However, this opportunity is not available in most foreign jurisdictions. As a result, defensive publications have their most predictable effect in the destruction of non-US patent rights.

\section{When is publication a desirable strategy?}

When considering a defensive publication strategy, keep in mind that your defensive publication can be used against you as well as against your competitors. In the United States, when an inventor publishes his or her invention, a one-year grace period ensures that the inventor's patent rights are not immediately extinguished. Publication immediately extinguishes most non-US patent rights, however, even those of the inventor. A decision to publish is thus a decision to irreversibly surrender potential patent rights. Such an important decision should only be made in the context of a comprehensive IP strategy. Viewing the potential impact of your defensive publications in light of a high-level strategy will reduce the possibility of strategic mistakes that could damage your IP position.

In general, publication should be considered when (i) the cost of patenting outweighs the benefit of the patent monopoly and (ii) the invention cannot be protected in a manner that is sufficiently secure to support trade-secret protection. The flow chart shown in Figure 1 provides a useful framework for determining the disposition of any specific invention.

Trade-secret versus patent protection Trade secrets protect against misappropriation of inventions that are kept secret, and not against independent discovery or reverse engineering. An invention is therefore a good candidate for trade-secret protection when (i) the invention embodies a high degree of complexity and novelty that

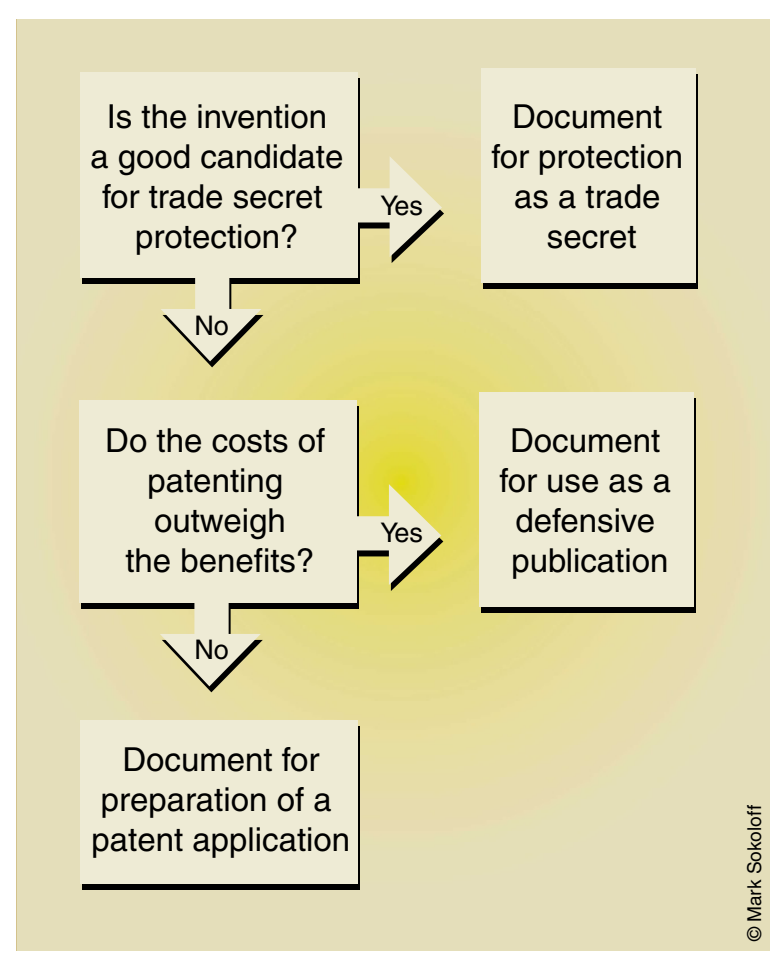

Figure 1. Flow chart showing the process for deciding whether an invention will be kept as a trade secret, patented, or made the subject of a defensive publication. 
would make independent invention by a competitor unlikely; (ii) the novel aspects of the invention are not embodied in a form that would permit the invention to be reverse engineered; and (iii) the invention is so securely protected that it is not likely to walk out the door with a customer or an ex-employee.

The classic example of a good candidate for trade-secret protection is a breakthrough manufacturing process that cannot be deduced from the product manufactured. For example, a discovery that a previously patented drug can be synthesized more efficiently using an unusual set of reagents and reaction conditions is a good candidate for trade-secret protection. Patenting of such a process would require disclosure of the invention to the public, and the risk of infringement might not provide sufficient deterrent to prevent others from secretly using the process. Nevertheless, such an invention should be maintained as a trade secret only if the company is committed to taking the stringent security measures necessary to maintain the secrecy of the invention.

In determining whether to maintain an invention as a trade secret, it is important not to stumble over the "best mode" requirement. Patent applicants often like the idea of patenting a basic invention while retaining detailed information about some special aspect of the invention, such as a superior method of making it. However, the patent law requires the patent application to describe the best mode subjectively known to the inventor for carrying out his or her invention ${ }^{2}$. Failure to include the best mode can result in the invalidation of the resulting patent; thus, patent applicants should not attempt to retain as a trade secret the best mode while seeking a patent on other, less effective modes of carrying out the invention. In some circumstances, an opportunity may exist to patent certain aspects of an invention while retaining other aspects as trade secrets. However, decisions about such approaches should be made in light of a comprehensive strategy in consultation with a patent attorney or agent who has been fully informed of all aspects of the invention.

If the invention is not a good candidate for trade-secret protection, the next step is to consider whether patent protection is warranted. Patent protection is generally preferable to publication, especially for the following categories of inventions: core or platform technologies with multiple applications; improvements to core technologies where the core technology is not already protected by patent; and improvements to patented inventions where patent term is important.
Invention of a core technology with multiple commercial applications generally provides the foundation for broad patent protection. For example, discovery, expression, and sequencing of a gene associated with a disease is usually sufficient to obtain a patent covering the isolated gene, the synthetically produced gene, degenerate versions of the gene, the gene as a component of a heterologous DNA sequence, the gene as a component of a plasmid, a cell comprising the plasmid, and so on. For a commercially important gene, this potentially broad patent coverage is rarely outweighed by the costs of patenting. Moreover, because genetic inventions are often subject to reverse engineering and independent discovery, trade-secret protection may not be adequate. Consequently, a patent is the preferred form of protection for such platform inventions.

Improvements to core technologies not already protected by patents often form the basis of narrow, but valuable, patent protection. For example, consider the situation in which a gene is publicly available and therefore not patentable. A novel mutation that results in an improvement in the gene product may open the door to narrow patent protection for a nucleic acid with the improved nucleotide sequence. Patent protection of narrow, incremental improvements is especially preferred when the patent's claims will encompass a commercial product. If, for example, the mutated gene will form a component of your product, patenting is the preferred mode of protection. On the other hand, if the incremental invention (i) will not be embodied in a commercial product (e.g., the mutated gene results in a product that is not as good as the current lead product) and (ii) is already embodied in the broad claims of your patent portfolio, then publication to prevent patenting by a competitor may be warranted.

Where an invention has potential longterm marketability, patenting incremental inventions embodied in products is advisable, even when an existing patent already protects the invention. In such circumstances, patenting of incremental inventions can help to extend the life of the patent coverage for the product. Pharmaceutical companies often patent incremental inventions to extend the length of patent coverage for a drug product. Such incremental inventions typically include novel formulations, new methods of administration, specialized drug delivery devices, improved dosage regimens, and dosage forms combining more than one active ingredient. Moreover, for high- revenue products, it may be desirable to patent incremental inventions even when the incremental inventions are not embodied in a product, especially if the incremental invention is an alternative that may be a truly effective competitor.

Defensive publication should be preferred over patent protection only if the cost of obtaining a patent is not justified by the value of the patent monopoly. The classic scenario for use of defensive publications involves incremental inventions that are (i) covered by existing patent claims and (ii) not embodied in a product with potentially long-term marketability. Consider the example of a novel gene. If a strong patent portfolio exists with claims broadly covering the gene and methods of making the gene product using bacteria from the Salmonella genus, new data that yet another Salmonella species produces a properly folded gene product would be a good candidate for publication. If the invention is not covered by existing claims, then publishing the invention only assists competitors in practicing the invention. If the invention is embodied in a product with long-term marketability, then the expense of obtaining patent coverage is likely to be justified by the extended patent protection.

Other examples of potentially effective publications include the following:

Uses of a core technology that are not strong candidates for patent protection. Even though they are not strong candidates, a competitor may convince the patent office to issue a patent. An issued patent, even if it is invalid, is entitled to a presumption of validity, and expensive litigation may be required to overcome this presumption.

Inventions in filed applications. Most countries automatically publish patent applications 18 months after the initial filing. This results in an 18-month window during which a non-US patent application can be filed in a foreign country. Early publication of your own patent application (i.e., before the 18-month publication) can prevent this. Keep in mind, however, that the early publication can be used against your own subsequently filed non-US patent applications as well.

Inventions in catalogs or other advertising material. Catalogs, web sites, and other customer information all qualify as prior art. However, these items often are not searched by the patent offices because they are not in searchable databases. Publication of such materials in a forum that is readily searchable by patent examiners can increase the probability that examiners will find these publications and use them against your competitor's patents. 


\section{How to write a defensive publication}

The probability of executing a successful defensive publication strategy is vastly improved if the defensive publication strategy is part of a comprehensive patent strategy. As noted above, defensive publications relating to important company technology are best employed only where the company already has effective patent protection. For this reason, it is often necessary to analyze the claims of your existing patents before publishing to be sure that they encompass the alternatives being described in your defensive publication. In the absence of such protection, your defensive publication may simply assist your competitor in engineering around your patent. Regularly scheduled reviews can be used to identify and evaluate the value of each new invention, so that a decision can be made regarding whether to patent, publish, or maintain the invention as a trade secret. If a decision is made to publish, the form and content of the defensive publication should be carefully considered.

According to the US Federal courts, the impact of a publication on patentability is controlled by the "enablement requirement." To knock out a patent application, a publication must "enable" the invention claimed in the patent application: that is, the publication must "disclose every element of the challenged claim and enable one skilled in the art to make the anticipating subject matter" 3 . An assessment of the probable value of a defensive publication should take into account that the publication will not prevent others from patenting aspects of the invention that are not enabled by the publication. In complex fields like biotechnology, where the efficacy of conceptual inventions is viewed as unpredictable, this application of the enablement requirement means that the defensive publication should provide a detailed protocol for obtaining the invention and should generally describe empirical work demonstrating the validity of the inventive concept.

For example, consider the publication of a new gene sequence. Publication of the sequence enables an ordinary molecular biologist to make a nucleic acid having the exact sequence of the gene. Publication of the sequence may not, however, enable an ordinary molecular biologist to use the gene in a specific gene-therapy application. The publication would destroy the right of others to patent the isolated or synthetically produced nucleic acid encoding the gene, but may not destroy the patent rights of one who later succeeds in using the nucleic acid in an unpredictable gene-therapy application.

To strengthen the impact of the publica- tion, consider including alternatives to the invention. Including alternatives to the invention will protect against competitors who wish to engineer around the published invention to generate patentable alternatives. For example, if the defensive publication includes a gene sequence, consider including language quantifying the permissible amount of variation in the sequence-that is, the amount of variation that can be tolerated without destroying the activity of the gene product. Your patent counsel can assist you in broadening the impact of a defensive publication by providing language commonly used in

\section{A tempting forum for defensive publishing is the standard company web site. The content of web sites is constantly changing, however, and most do not include a method of verifying the date and authenticity of the publication.}

patents to broaden the scope of an invention. Broadening the defensive publication in this manner will reduce the risk that others will patent related inventions; however, surprising improvements or alternatives may still be patentable. As you broaden the scope, consider that you may need to describe experiments supporting the enablement of those alternatives.

\section{Where to publish}

Arenas for publishing defensive publications range from traditional peer-reviewed journals to online publications. Publication in peer-reviewed journals is a desirable option because the critical review afforded such publications validates their contents and is important to the advancement of science. The traditional requirement of repeatability in peer-reviewed publications parallels the enablement requirement, helping to ensure that the latter is satisfied. The difficulty with traditional journal publications is that submitted articles can take months to be published. Consequently, if timing is important to the patent strategy, refereed journals should not be relied on for defensive publishing purposes.

Before 1998, IBM maintained its own defensive publishing bulletin, the IBM Technical Disclosure Bulletin. In 1998, IBM began publishing its technical disclosures in Research Disclosure, a defensive publishing forum published by Emsworth Design, Inc. since the 1960s. Research Disclosure is a World Intellectual Property Organizationrecommended journal, which helps to ensure that it is on the radar of patent examiners around the world. More than 1,000 companies use Research Disclosure, which publishes about 400 disclosures a month, and Emsworth plans to begin accepting publications through the Internet in the near future.

A tempting forum for defensive publishing is the standard company web site. The content of web sites is constantly changing, however, and most do not include a method of verifying the date and authenticity of the publication. Additionally, webbased searching is still not as accurate as searching of traditional information databases. These problems reduce the probability that a web site publication will be identified and considered by patent examiners.

IP.com is an Internet-based defensive publishing company that seeks to address current online publication problems. IP.com's system electronically date-stamps and authenticates each defensive publication to ensure that its publications have legal significance for the world's patent systems. Documents published by IP.com become part of the text-searchable database accessible to the world's patent offices. The disclosures are also published monthly in The IP.com Journal to ensure compliance with accepted legal standards.

\section{Conclusions}

The word "publish" often brings fear into the hearts of patent attorneys, and rightly so. In sectors such as the biotechnology and pharmaceutical industries, intellectual capital is king. Patents are a prerequisite to protecting some of the most valuable aspects of that intellectual capital and are thus a key to competitive survival. Ill-considered and untimely publications can be patent poison. Yet, like any poison, publication can also be used as a weapon. In attempting to avoid damaging their own patent potential through poorly timed publication, companies and academic institutions should take care not to overlook the defensive use of publications. A successfully executed defensive publication strategy can, without the need for expensive patent prosecution, help secure freedom to operate by preventing others from patenting in the technology space described in the publication.

1. IBM Technical Disclosure Bulletin (http://www.ibm. com/ibm/licensing/ibm_tdb/).

2. 35 USC $\S 112$

3. PPG Industries, Inc. v. Guardian Industries, Corp., 37 U.S.P.Q.2d 1618 (Fed. Cir. 1996). 